\title{
Synthesis and X-ray crystal structures of zinc dichloride complexes supported by a $\beta$-diimine ligand
}

\author{
Christoph Scheiper, Dominik Naglav, Dieter Bläser, Christoph Wölper, Stephan Schulz*
}

Keywords: Zinc, $\beta$-Iminoamines, $\beta$-Diimine Complexes, Tautomerism

$\beta$-diimine zinc dichloride complexes $\left[\mathrm{CH}_{2}\{\mathrm{C}(\mathrm{Me}) \mathrm{NAr}\}_{2}\right] \mathrm{ZnCl}_{2}(\mathrm{Ar}$ $=$ Mes 1, Dipp 2) were obtained from the reactions of $\mathrm{ZnCl}_{2}$ with the corresponding $\beta$-iminoamines $[\mathrm{ArN}(\mathrm{H}) \mathrm{C}(\mathrm{Me}) \mathrm{CHC}(\mathrm{Me}) \mathrm{NAr}] .1$ and $\mathbf{2}$ were characterized by multinuclear NMR $\left({ }^{1} \mathrm{H},{ }^{13} \mathrm{C}\right)$ and IR

spectroscopy, elemental analyses as well as by single crystal Xray diffraction. The energy differences between the enamineimine tautomers of the $\beta$-iminoamines were quantified by quantum chemical calculations.

\footnotetext{
* Prof. Dr. Stephan Schulz

Fax: + 49 0201-1834635, Phone: + 4902011834635

stephan.schulz@uni-due.de

Faculty of Chemistry, University of Duisburg-Essen, 45117

Essen, Germany

E-mail: Homepage: http:/www.uni-due.de/ak schulz/

$\square$ Supporting information for this article is available on the

WWW under http://dx.doi.org/10.1002/zaac.201000xxx or from the author.
}

\section{Introduction}

Monoanionic $\beta$-diketiminate ligands, often also referred to as "nacnac" ligands, have been established over the last decades as powerful spectator ligands for the stabilization of main group and transition metal complexes as well as lanthanide complexes. ${ }^{[1-3]}$ According to the easy tunability of the steric and electronic properties of these ligands as well as their variable binding motifs, ${ }^{[4]}$ which range from pure $\sigma$ to a combination of $\sigma$ and $\pi$ donation, they have evolved into one of the most ubiquitous systems in coordination chemistry. The kinetic stabilization of lowcoordinated metal complexes, in which the metal centers adopt low formal oxidation states, by use of sterically very demanding $\beta$-diketiminate ligands has been of particular interest. ${ }^{[5-9]}$ Moreover, $\beta$-diketiminate metal complexes are promising catalyst in different catalytic reactions such as ring opening polymerization (ROP) of cyclic ethers and others. ${ }^{[10-13]}$

While a large number of metal complexes containing monoanionic $\beta$-diketiminate ligands have been explored, complexes containing the neutral $\beta$-iminoamine $(\beta$ diketimine) ligands are rather rare, whereas $\alpha$-diimines complexes, which have been established over the last decade as very powerful catalysts (Brookhart-type), are well known. ${ }^{[14-17]} \beta$-diketimines can formally exist in the enamine and the imine form, but the enamine tautomer is typically energetically favoured (scheme 1). However, the $\beta$-diimine tautomer can be stabilized by use of sterically demanding substituents as was successfully demonstrated in the synthesis of the bulky diimine $\mathrm{CH}_{2}[\mathrm{C}(t-\mathrm{Bu}) \mathrm{NDipp}]_{2}(\mathrm{Dipp}=$ $\left.\left.2,6-i-\operatorname{Pr}_{2} \mathrm{C}_{6} \mathrm{H}_{3}\right\}_{2}\right)$, which does not interconvert in hydrocarbon solutions to the enamine form $[\mathrm{DippN}(\mathrm{H}) \mathrm{C}(t$ $\mathrm{Bu}) \mathrm{CHC}(t-\mathrm{Bu}) \mathrm{NDipp}]^{[18]}$
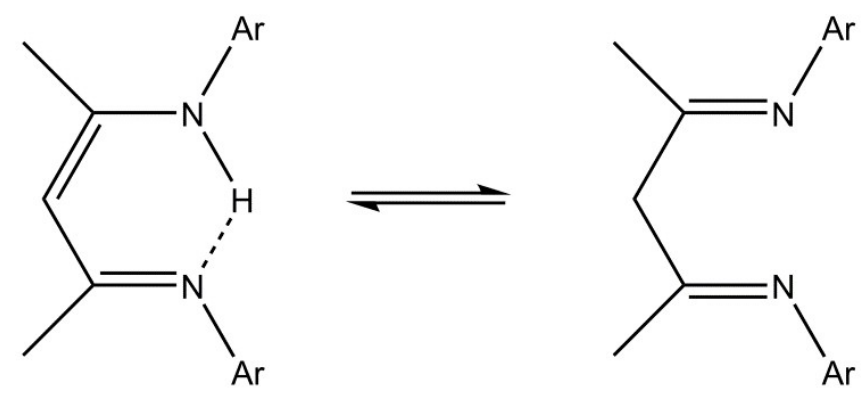

Scheme 1. Enamine-imine tautomers of $\beta$-iminoamine.

Moreover, the $\beta$-diimine form can be stabilized in the coordination sphere of transition metals as was initially shown for the nickel complex $\left[\mathrm{CH}_{2}(\mathrm{C}(\mathrm{Me}) \mathrm{NDipp})_{2}\right] \mathrm{NiBr}_{2}$, which was obtained from the reaction of $\mathrm{NiBr}_{2}$ with the $\beta$ iminoamine $[\operatorname{DippN}(\mathrm{H}) \mathrm{C}(\mathrm{Me}) \mathrm{CHC}(\mathrm{Me}) \mathrm{NDipp}] .{ }^{[19]}$ Since then, a few neutral ${ }^{[20-23]}$ as well as cationic metal complexes $^{[24-26]}$ of the desired type have been synthesized and structurally characterized (scheme 2).
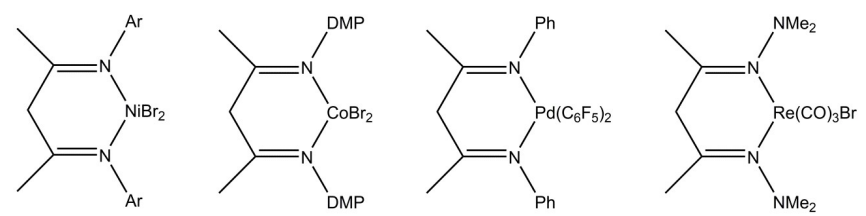

$\mathrm{Ar}=\mathrm{Ph}, \mathrm{o}-\mathrm{CH}_{3}-\mathrm{C}_{6} \mathrm{H}_{4}, \mathrm{Mes}$, Dipp; DMP = 2,6- $-\mathrm{Me}_{2}-\mathrm{C}_{6} \mathrm{H}_{3}$

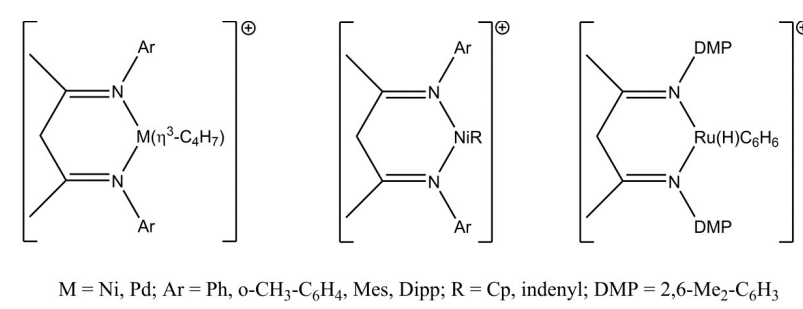

Scheme 2. Structurally characterized neutral and cationic $\beta$ diimine transition metal complexes. 
We herein report on the synthesis and solid state structures of first $\beta$-diimine zinc dichloride complexes $\left[\mathrm{CH}_{2}\{\mathrm{C}(\mathrm{Me}) \mathrm{NAr}\}_{2}\right] \mathrm{ZnCl}_{2}(\mathrm{Ar}=\mathrm{Mes}$ 1, Dipp 2).

\section{Results and Discussion}

Reactions of $\mathrm{ZnCl}_{2}$ with equimolar amounts of the $\beta$ iminoamines $[\mathrm{ArN}(\mathrm{H}) \mathrm{C}(\mathrm{Me}) \mathrm{CHC}(\mathrm{Me}) \mathrm{NAr}]$ proceed with formation of the corresponding $\beta$-diimine complexes $\left[\mathrm{CH}_{2}\left\{\mathrm{C}(\mathrm{Me}) \mathrm{NAr}_{2}\right] \mathrm{ZnCl}_{2}(\mathrm{Ar}=\operatorname{Mes} \mathbf{1}\right.$, Dipp 2). 1 and 2 were characterized by multinuclear NMR $\left({ }^{1} \mathrm{H},{ }^{13} \mathrm{C}\right)$ and IR spectroscopy as well as by elemental analysis. ${ }^{[27]}$ In addition, the solid state structure of $\mathbf{1}$ and $\mathbf{2}$ were determined by single crystal X-ray diffraction.
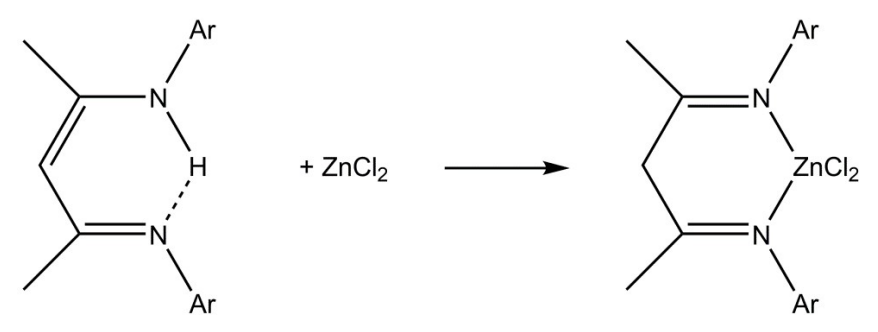

$\operatorname{Ar}=$ Mes 1, Dipp 2

Scheme 3. Synthesis of $\mathbf{1}$ and 2.

Compared to the starting $\beta$-iminoamines, the ${ }^{1} \mathrm{H}$ NMR spectra of $\mathbf{1}$ and $\mathbf{2}$ show a resonances for the methylene group and the C-Me groups with a relative intensity of 2:6, while the resonances of the $\mathrm{N}-\mathrm{H}$ protons $(\mathrm{R}=$ Mes 12.08 ; Dipp $12.07 \mathrm{ppm}$ ) have disappeared. In addition, the $\gamma-\mathrm{CH}$ backbone resonances in the ${ }^{1} \mathrm{H}$ NMR spectra of the $\beta$ diketimines (Mes 4.91; Dipp 4.94 ppm) are shifted significantly to higher field (1: 4.20, 2: $4.34 \mathrm{ppm})$, indicating the formation of a methylene group $\left(\gamma-\mathrm{CH}_{2}\right)$. This was further proven by ${ }^{13} \mathrm{C}$ NMR spectroscopy, which also showed a significant highfield shift of the $\gamma-\mathrm{C}$ resonance (1: 44.9, 2: $45.1 \mathrm{ppm}$ ) compared to the $\beta$-iminoamines (Mes 93.9; Dipp 93.3 ppm). Moreover, the dept-135 ${ }^{13} \mathrm{C}$ NMR spectra show a negative signal, verifying the formation of a $\gamma-\mathrm{CH}_{2}$ group. The presence of the imine functionality was also proven by IR spectroscopy. IR spectra of $\mathbf{1}$ and $\mathbf{2}$ show strong absorption bands at 1659 (1) and $1656 \mathrm{~cm}^{-1}(2)$ due to the $\mathrm{N}=\mathrm{C}$ group.

Crystals of $\mathbf{1}$ and $\mathbf{2}$ suitable for a single crystal X-ray diffraction study were obtained from solutions in $\mathrm{CHCl}_{3} \mathbf{1}$ and THF 2 after storage at ambient temperature for $24 \mathrm{~h}$.

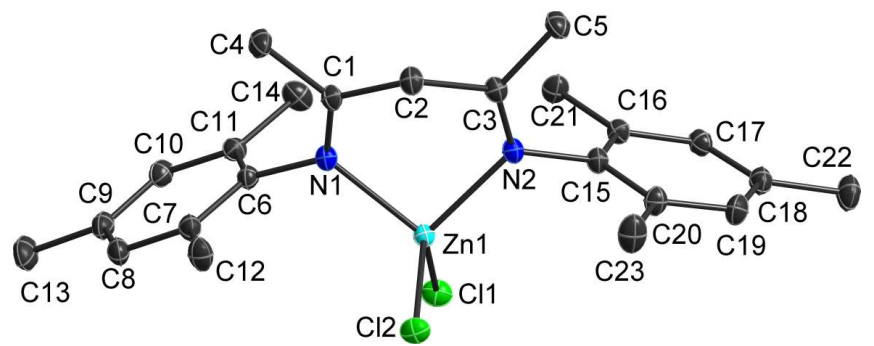

Figure 1. Molecular structure of $\mathbf{1}$ (displacement parameters are drawn at $50 \%$ probability level, solvent molecules and $\mathrm{H}$ atoms omitted) showing the atom-numbering scheme; selected bond lengths $(\AA)$ and angles $\left(^{\circ}\right)$ : Zn1-Cl1 2.2095(2), Zn1-Cl2 2.2528(2), Zn1-N1 2.0328(7), Zn1-N2 2.0351(7), C1-C2 1.5119(12), C2-C3 1.5101(11), N1-C1 1.2836(10), N2-C3 1.2851(10); N1-Zn1-N2 95.27(3), Cl1-Zn1-Cl2 (112.678(9), C1-C2-C3 121.90(7).

Table 1. Crystallographic Data and Measurements for $\mathbf{1}$ and 2.

\begin{tabular}{|c|c|c|}
\hline & 1 & 2 \\
\hline $\begin{array}{l}\text { Empirical formula } \\
M\end{array}$ & $\begin{array}{l}\mathrm{C}_{25} \mathrm{H}_{32} \mathrm{Cl}_{8} \mathrm{~N}_{2} \mathrm{Zn} \\
709.52\end{array}$ & $\begin{array}{l}\mathrm{C}_{29} \mathrm{H}_{42} \mathrm{Cl}_{2} \mathrm{~N}_{2} \mathrm{Zn} \\
554.91\end{array}$ \\
\hline Crystal size $[\mathrm{mm}]$ & $0.5 \times 0.5 \times 0.45$ & $\begin{array}{l}0.362 \times 0.279 \times \\
0.138\end{array}$ \\
\hline$T[\mathrm{~K}]$ & $100(1)$ & $100(1)$ \\
\hline Crystal system & monoclinic & monoclinic \\
\hline Space group & $P 2{ }_{1} / n$ & $P 2{ }_{1} / c$ \\
\hline$a[\AA]$ & $13.3781(8)$ & $13.3723(7)$ \\
\hline$b[\AA]$ & $13.2265(8)$ & $16.1334(8)$ \\
\hline$c[\AA]$ & $18.1080(12)$ & $14.9812(7)$ \\
\hline$\alpha\left[^{\circ}\right]$ & 90 & 90 \\
\hline$\beta\left[^{\circ}\right]$ & $101.614(3)$ & $110.875(2)$ \\
\hline$\gamma\left[{ }^{\circ}\right]$ & 90 & 90 \\
\hline$V\left[\AA^{3}\right]$ & $3138.5(3)$ & $3019.9(3)$ \\
\hline$Z$ & 4 & 4 \\
\hline$D_{\text {calc }}\left[\mathrm{g} \cdot \mathrm{cm}^{-1}\right]$ & 1.502 & 1.221 \\
\hline$\mu\left(\mathrm{MoK}_{\alpha}\left[\mathrm{mm}^{-1}\right]\right)$ & 1.482 & 1.009 \\
\hline Transmissions & $0.75 / 0.66$ & $1.0 / 0.8721$ \\
\hline$F(000)$ & 1448 & 1176 \\
\hline \multirow[t]{3}{*}{ Index ranges } & $-21 \leq h \leq 20$ & $-20 \leq h \leq 20$ \\
\hline & $-21 \leq k \leq 21$ & $-24 \leq k \leq 24$ \\
\hline & $-28 \leq l \leq 29$ & $-21 \leq l \leq 23$ \\
\hline$\theta_{\max }\left[{ }^{\circ}\right]$ & 36.24 & 33.197 \\
\hline $\begin{array}{l}\text { Reflections } \\
\text { collected }\end{array}$ & 70900 & 136502 \\
\hline $\begin{array}{l}\text { Independent } \\
\text { reflections }\end{array}$ & 14035 & 11549 \\
\hline$R_{\text {int }}$ & 0.0210 & 0.0285 \\
\hline Refined parameters & 333 & 317 \\
\hline$R_{1}[I>2 \sigma(I)]$ & 0.0241 & 0.0304 \\
\hline$w R_{2}$ [all data] & 0.0661 & 0.0827 \\
\hline GooF & 1.031 & 1.022 \\
\hline $\begin{array}{l}\Delta \rho_{\text {final }}(\max / \min )[\mathrm{e} \\
\left.\AA^{-3}\right]\end{array}$ & $1.218 /-0.801$ & $0.938 /-0.500$ \\
\hline
\end{tabular}

${ }^{[\mathrm{a}]} R 1=\Sigma\left(|| F_{\mathrm{o}}|-| F_{\mathrm{c}}||\right) / \Sigma\left|F_{\mathrm{o}}\right|($ for I $>2 \sigma(\mathrm{I})){ }^{[\mathrm{b}]} w R 2=\left\{\Sigma\left[w\left(F_{\mathrm{o}}{ }^{2}-\right.\right.\right.$ $\left.\left.F_{\mathrm{c}^{2}}^{2}\right] / \Sigma\left[w\left(F_{\mathrm{o}}^{2}\right)^{2}\right]\right\}^{1 / 2}$. [c] Goodness of fit $=\left\{\Sigma\left[w\left(\left|F_{\mathrm{o}}^{2}\right|-\right.\right.\right.$ $\left.\left.\left.\left|\mathrm{F}_{\mathrm{c}}{ }^{2}\right|\right)^{2}\right] /\left(\mathrm{N}_{\text {observns }}-\mathrm{N}_{\text {params }}\right)\right\}^{1 / 2}$

1 crystallizes in the monoclinic space group $P 2_{1} / n$ with one molecule in the asymmetric unit accompanied by two chloroform molecules. 2 crystallizes in space group $P 2_{1} / c$ without any solvent. The central six-membered, nonplanar $\mathrm{C}_{3} \mathrm{~N}_{2} \mathrm{Zn}$ rings in $\mathbf{1}$ and $\mathbf{2}$ adopt boat-type conformations as was previously reported for this type of complexes, resulting from the protonation of the backbone carbon atom $(\gamma-\mathrm{C})$. The fourfold-coordinated $\mathrm{Zn} 1$ atom, which adopts a distorted tetrahedral coordination sphere, and the $\mathrm{C} 2$ atom are slightly out of the plane (C2: $0.4062(12) \AA$, Zn: $0.5216(11) \AA$ (1); C2: 0.4826(19) $\AA, \mathrm{Zn}: 0.7400(15) \AA(2))$ defined by N1, N2, C1 and C3. The endocyclic N1-Zn1-N2 bond angles $\left(\mathbf{1}: 95.27(3)^{\circ}, \mathbf{2}\right.$ : $\left.93.13(3)^{\circ}\right)$ are significantly smaller than the exocyclic $\mathrm{Cl1}-\mathrm{Zn} 1-\mathrm{Cl} 2$ bond angle (1: 112.678(9) ${ }^{\circ}, 2$ : $\left.118.128(17)^{\circ}\right)$. The endocyclic C1-C2-C3 
bond angles of $121.90(7)^{\circ}$ in $\mathbf{1}$ and $119.76(10)^{\circ}$ in $\mathbf{2}$ are significantly larger than the ideal tetrahedral value of a $\mathrm{sp}^{3}$ hybridized carbon atom. The $\mathrm{N} 1$ and $\mathrm{N} 2$ atoms are trigonal planar ( $\Sigma$ of the bond angles 1: $359.7^{\circ}, 2: 359.84(\mathrm{~N} 1)$ and 1: $359.9^{\circ}, 2: 358.96(\mathrm{~N} 2)$ ), which is fully consistent with the description of $\mathrm{sp}^{2}$-hybridized (imine) $\mathrm{N}$ atoms. The $\mathrm{Zn}-\mathrm{Cl}$ bond lengths in $\mathbf{1}$ differ by almost $5 \mathrm{pm}$ (2.2095(2), $2.2528(2) \AA$ ) and in 2 by only 3 pm (2.1979(3), 2.2212(4) $\AA)$. The $\mathrm{Zn}-\mathrm{N}$ bond lengths in $\mathbf{2}$ are slightly elongated compared to those in $\mathbf{1}$ (1: 2.0328(7), 2.0351(7) $\AA$; 2 : $2.0515(8), 2.0515(8) \AA)$, reflecting the larger steric demand of the Dipp substituents. The $\mathrm{Zn}-\mathrm{N}$ bond lengths in $\mathbf{1}$ and $\mathbf{2}$ are elongated by almost $6 \mathrm{pm}$ compared to the average $\mathrm{Zn}-\mathrm{N}$ bond lengths reported in the Cambridge structure database for 23 structurally characterized neutral $\beta$-diketiminate-zinc complexes containing the analogous Mes-substituted ligand (58 $\mathrm{Zn}-\mathrm{N}$ bonds, mean value $1.977(3)$, median value 1.971 $\AA) .{ }^{[28-40]}$ The C1-C2 (1: 1.5119(12) $\left.\AA ; 2: 1.5163(16) \AA\right)$ and C2-C3 (1: 1.5101(11) $\AA$; 2: 1.5151(15) $\AA$ ) bond lengths as well as the N1-C1 (1: $1.2836(10) \AA ; 2: 1.2802(14) \AA)$ and

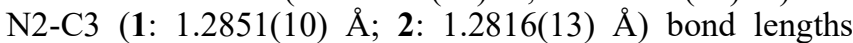
agree perfectly with the description of $\mathrm{C}-\mathrm{C}$ single bonds and $\mathrm{C}-\mathrm{N}$ double bonds.

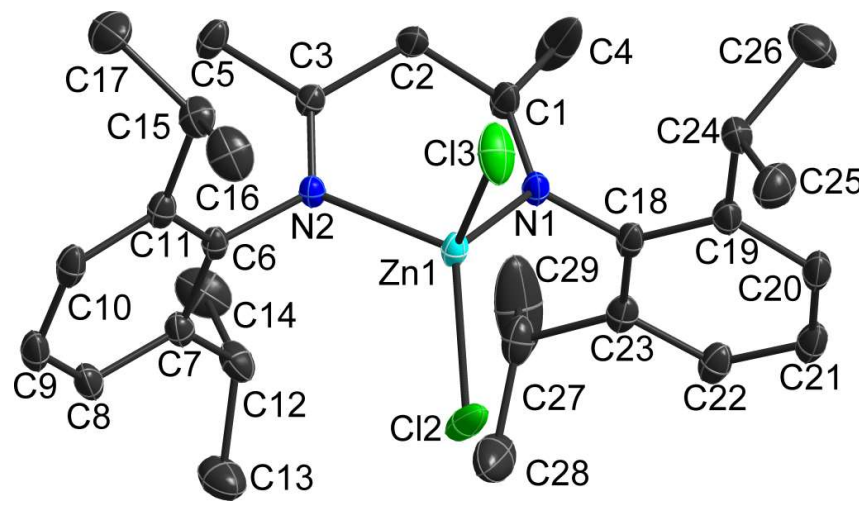

Figure 2. Molecular structure of $\mathbf{2}$ (displacement parameters are drawn at $50 \%$ probability level, $\mathrm{H}$ atoms omitted) showing the atom-numbering scheme; selected bond lengths $(\AA)$ and angles $\left(^{\circ}\right)$ : $\mathrm{Zn}(1)-\mathrm{N}(2) \quad 2.0515(8), \quad \mathrm{Zn}(1)-\mathrm{N}(1) \quad 2.0515(8), \quad \mathrm{Zn}(1)-\mathrm{Cl}(2)$ 2.1979(3), $\mathrm{Zn}(1)-\mathrm{Cl}(3) 2.2212(4), \mathrm{C}(1)-\mathrm{C}(2) 1.5163(16), \mathrm{C}(2)-\mathrm{C}(3)$ $1.5151(15), \mathrm{N}(1)-\mathrm{C}(1) 1.2802(14), \mathrm{N}(2)-\mathrm{C}(3) 1.2816(13), \mathrm{N}(2)-$ $\mathrm{Zn}(1)-\mathrm{N}(1)$ 93.13(3), $\mathrm{Cl}(2)-\mathrm{Zn}(1)-\mathrm{Cl}(3)$ 118.128(17), C(3)-C(2)C(1) $119.76(10)$.

Since there is no experimental evidence that the $\beta$ aminoamines $[\mathrm{ArN}(\mathrm{H}) \mathrm{C}(\mathrm{Me}) \mathrm{CHC}(\mathrm{Me}) \mathrm{NAr}]$ form an equilibrium with the corresponding $\beta$-diimine tautomers in solution, we investigated the relative energies of both tautomers using quantum chemical calculations. ${ }^{[41]}$ Geometry optimization of both tautomeric forms clearly revealed that both molecules $[\mathrm{ArN}(\mathrm{H}) \mathrm{C}(\mathrm{Me}) \mathrm{CHC}(\mathrm{Me}) \mathrm{NAr}]$ strongly prefer the enamine form by $31.51(\mathrm{Ar}=$ Mes 1a) and $33.68 \mathrm{~kJ} / \mathrm{mol}(\mathrm{Ar}=\operatorname{Dipp~2a})$, respectively. Therefore, the formation of the $\beta$-diimine complexes 1 and 2 must be initiated by the coordination of the $\beta$-iminoamines to the electrophilic $\mathrm{ZnCl}_{2}$, followed by proton shift to the nucleophilic $\gamma$-carbon of the ligand backbone.
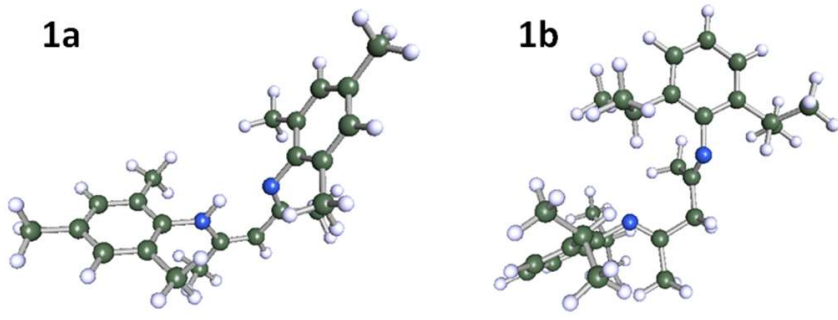

Figure 3. Geometry-optimized structures of both tautomeric forms of $[\mathrm{MesN}(\mathrm{H}) \mathrm{C}(\mathrm{Me}) \mathrm{CHC}(\mathrm{Me}) \mathrm{NMes}]$ (enamine 1a, imine 1b).

\section{Conclusions}

The syntheses of $\beta$-diimine zinc dichloride complexes $\left[\mathrm{CH}_{2}\{\mathrm{C}(\mathrm{Me}) \mathrm{NAr}\}_{2}\right] \mathrm{ZnCl}_{2}(\mathrm{Ar}=$ Mes 1, Dipp 2) is described and the solid state structures of $\mathbf{1}$ and $\mathbf{2}$ are determined. According to quantum chemical calculations, the enamine tautomeric forms of the $\beta$-iminoamines [ArN(H)C(Me)CHC(Me)NAr] $(\mathrm{Ar}=$ Mes, Dipp $)$ are preferred by $31.51 \mathrm{~kJ} / \mathrm{mol}(\mathrm{Ar}=\mathrm{Mes})$ and $33.68 \mathrm{~kJ} / \mathrm{mol}(\mathrm{Ar}$ $=$ Dipp). Obviously, the formation of the $\beta$-diimine tautomers in $\mathbf{1}$ and $\mathbf{2}$ is initiated by coordination of the $\beta$ iminoamines to the electrophilic zinc dichloride.

\section{Experimental Section}

All manipulations were performed in a glovebox under $\mathrm{N}_{2}$ atmosphere or by standard Schlenk techniques. $\mathrm{L}^{1 / 2} \mathrm{H}\left(\mathrm{L}^{1}=\right.$ $[\mathrm{MesN}(\mathrm{H}) \mathrm{C}(\mathrm{Me}) \mathrm{CHC}(\mathrm{Me}) \mathrm{NMes}], \mathrm{L}^{2}=[\operatorname{DippN}(\mathrm{H}) \mathrm{C}(\mathrm{Me}) \mathrm{CHC}-$ (Me)NDipp]) were prepared according to literature methods. ${ }^{[22]}{ }^{1} \mathrm{H}$ and ${ }^{13} \mathrm{C}\left\{{ }^{1} \mathrm{H}\right\}$ spectra were recorded using a Bruker AMX 300 spectrometer and are referenced to internal $\mathrm{CD}_{2} \mathrm{Cl}_{2}\left(\delta^{1} \mathrm{H} 5.32, \delta^{13} \mathrm{C}\right.$ 53.8). IR spectra were recorded on an Alpha-T FT-IR spectrometer with a single reflection ATR sampling module. Melting points were measured in sealed capillaries and were not corrected. Elemental analyses were performed at the Elementaranalyse Labor of the University of Duisburg-Essen.

General Preparation of $\left[\mathrm{CH}_{2}\{\mathrm{C}(\mathrm{Me}) \mathrm{NAr}\}_{2}\right] \mathrm{ZnCl}_{2}$.

0 A solution of $2.0 \mathrm{mmoL} \mathrm{L} \mathrm{L}{ }^{1 / 2} \mathrm{H}\left(\mathrm{L}^{1}=\right.$

$[\mathrm{MesN}(\mathrm{H}) \mathrm{C}(\mathrm{Me}) \mathrm{CHC}(\mathrm{Me}) \mathrm{NMes}] 0.67 \mathrm{~g} ; \mathrm{L}^{2}=$

[DippN(H)C(Me)CHC(Me)NDipp] $0.84 \mathrm{~g})$ and $0.27 \mathrm{~g} \mathrm{ZnCl}_{2}$ $(2.0 \mathrm{mmoL})$ in $10 \mathrm{~mL}$ of $\mathrm{CHCl}_{3}$ was stirred for $1 \mathrm{~h}$ at ambient temperature. The solution was stored for $24 \mathrm{~h}$, yielding colorless crystals of $\mathbf{1}$, whereas 2 crystallized in THF.

$\left[\mathbf{C H}_{2}\left\{\mathbf{C}(\mathbf{M e}) \mathbf{N M e s}_{2}\right] \mathbf{Z n C l}_{2}\right.$ (1). Yield $0.88 \mathrm{~g}(93 \%)$; m.p. $321^{\circ} \mathrm{C}$. Anal. Calc. for $\mathrm{C}_{23} \mathrm{H}_{30} \mathrm{~N}_{2} \mathrm{ZnCl}_{2}(470.78 \mathrm{~g} / \mathrm{mol}): \mathrm{C} 58.68, \mathrm{H} 6.42, \mathrm{~N}$ 5.95; Found: C 58.14, H 6.44, N 5.83\%. ${ }^{1} \mathrm{H}$ NMR (300 MHz, $\left.\mathrm{CD}_{2} \mathrm{Cl}_{2}, 25^{\circ} \mathrm{C}\right): \delta=2.11\left(\mathrm{~s}, 6 \mathrm{H}, \beta-\mathrm{CCH}_{3}\right), 2.21\left(\mathrm{~s}, 12 \mathrm{H}, o-\mathrm{CH}_{3}\right)$, $2.32\left(\mathrm{~s}, 6 \mathrm{H}, p-\mathrm{CH}_{3}\right), 4.20$ (s, $\left.2 \mathrm{H}, \gamma-\mathrm{CH}_{2}\right), 6.98(\mathrm{~s}, 4 \mathrm{H}, m-\mathrm{H})$. ${ }^{13} \mathrm{C}\left\{{ }^{1} \mathrm{H}\right\}$ NMR $\left(75 \mathrm{MHz}, \mathrm{CD}_{2} \mathrm{Cl}_{2}, 25{ }^{\circ} \mathrm{C}\right): \delta=18.6\left(o-\mathrm{CH}_{3}\right), 20.5$ $\left(\beta-\mathrm{CCH}_{3}\right), 24.7\left(p-\mathrm{CH}_{3}\right), 44.9(\gamma-\mathrm{C}), 128.6(m-\mathrm{C}), 129.4(o-\mathrm{C})$, $136.3(p-\mathrm{C}), 140.8(i-\mathrm{C}), 178.0\left(\beta-\mathrm{CCH}_{3}\right)$. ATR-IR: $v=2997$, 2986, 2954, 2914, 2870, 1659, 1631, 1476, 1428, 1385, 1207, 1188, $1139,1034,1014,856,825,722,606,549,436,403$.

$\left[\mathbf{C H}_{2}\{\mathbf{C}(\mathbf{M e}) \mathbf{N D i p p}\}_{2}\right] \mathbf{Z n C l}_{2}$ (2). Yield $0.99 \mathrm{~g}(89 \%) ;$ m.p. $256^{\circ} \mathrm{C}$. Anal. Calc. for $\mathrm{C}_{29} \mathrm{H}_{42} \mathrm{~N}_{2} \mathrm{ZnCl}_{2}(554.94 \mathrm{~g} / \mathrm{mol})$ : C 62.77, $\mathrm{H} 7.63, \mathrm{~N}$ 5.05; Found: C 62.41, H 7.34, N 5.17\%. ${ }^{1} \mathrm{H}$ NMR (300 MHz, $\left.\mathrm{CD}_{2} \mathrm{Cl}_{2}, 25^{\circ} \mathrm{C}\right): \delta=1.16\left(\mathrm{~d},{ }^{3} \mathrm{JHH}_{\mathrm{HH}}=6.8 \mathrm{~Hz}, 12 \mathrm{H}, \mathrm{CH}\left(\mathrm{CH}_{3}{ }^{1}\right)_{2}\right), 1.32$ $\left(\mathrm{d},{ }^{3} \mathrm{~J}_{\mathrm{HH}}=6.8 \mathrm{~Hz}, 12 \mathrm{H}, \mathrm{CH}\left(\mathrm{CH}_{3}{ }^{2}\right)_{2}\right), 2.12\left(\mathrm{~s}, 6 \mathrm{H}, \beta-\mathrm{CCH}_{3}\right), 3.03$ (sept, $\left.{ }^{3} J_{\mathrm{HH}}=6.8 \mathrm{~Hz}, 4 \mathrm{H}, \mathrm{CH}\left(\mathrm{CH}_{3}\right)_{2}\right), 4.34\left(\mathrm{~s}, 2 \mathrm{H}, \gamma-\mathrm{CH}_{2}\right), 7.28-$ 
$7.38(6 \mathrm{H}, m / p-\mathrm{H}) .{ }^{13} \mathrm{C} \mathrm{NMR}\left(75 \mathrm{MHz}, \mathrm{CD}_{2} \mathrm{Cl}_{2}, 25^{\circ} \mathrm{C}\right) \delta=24.0$ $\left(\mathrm{CH}\left(\mathrm{CH}_{3}{ }^{1}\right)_{2}\right), 24.7\left(\beta-\mathrm{CCH}_{3}\right), 26.3\left(\mathrm{CH}\left(\mathrm{CH}_{3}{ }^{2}\right)_{2}\right), 28.5\left(\mathrm{CH}\left(\mathrm{CH}_{3}\right)_{2}\right)$, $45.1(\gamma-\mathrm{C}), 124.5(m-\mathrm{C}), 127.5(p-\mathrm{C}), 139.9(o-\mathrm{C}), 140.4(i-\mathrm{C})$, $178.8\left(\beta-\mathrm{CCH}_{3}\right)$. ATR-IR: $v=3061,2964,2869,1656,1551$, 1463, 1441, 1383, 1260, 1096, 1017, 797, 752, 391.

Single crystal X-ray diffraction. Crystals of $\mathbf{1}$ and $\mathbf{2}$ were mounted on nylon loops in inert oil. Crystallographic data of $\mathbf{1}$ and $\mathbf{2}$ are summarized in Table 1. Figures 1 and 2 show the solid state structures of $\mathbf{1}$ and $\mathbf{2}$ including selected bond lengths and angles. Data were collected on a Bruker AXS D8 Kappa diffractometer with APEX2 detector (mono-chromated $\mathrm{Mo}_{K \alpha}$ radiation, $\lambda=$ $0.71073 \AA$ ) at $180(1) \mathrm{K}$. The structures were solved by Direct Methods (SHELXS-97) ${ }^{[43]}$ and refined anisotropically by fullmatrix least-squares on $F^{2}$ (SHELXL-97). ${ }^{[4]}$ Absorption corrections was performed semi-empirically from equivalent reflections on basis of multi-scans (Buker AXS APEX2, 1) and by face-indexing (2). All non-hydrogen atoms were refined anisotropically and hydrogen atoms were refined using a riding model or rigid methyl groups. The crystallographic data of $\mathbf{1}$ and $\mathbf{2}$ (excluding structure factors) have been deposited with the Cambridge Crystallographic Data Centre as supplementary publication no. CCDC-1035846 (1) and CCDC-1037909 (2).

Copies of the data can be obtained free of charge on application to CCDC, 12 Union Road, Cambridge, CB21EZ (fax: (+44)

1223/336033; e-mail: deposit@ccdc.cam-ak.uk).

Supporting Information (see footnote on the first page of this article): ${ }^{1} \mathrm{H}$ NMR, ${ }^{13} \mathrm{C}$ NMR and IR spectra of $\beta$-aminoimines and $\mathbf{1}$ and 2, X-ray crystallographic data of $\mathbf{1}$ and $\mathbf{2}$ incl. cif files as well as results from computational calculations.

\section{Acknowledgement}

Stephan Schulz gratefully acknowledges financial support by the University of Duisburg-Essen. We especially would like to thank M. Sc. Briac Tobey for his dedicated support and contributions concerning the theoretical calculations.

[1] L. Bourget-Merle, M. F. Lappert, J. S. Severn, Chem. Rev. 2002, 102, 3031-3066.

[2] Y.-C. Tsai, Coord. Chem. Rev. 2012, 256 722-758.

[3] S. P. Sarish, S. Nembenna, S. Nagendran, H. W. Roesky, Acc. Chem. Res. 2011, 44, 157-170.

[4] P. H. M. Budzelaar, A. B. Van Oort, A. G. Orpen, Eur. J. Inorg. Chem. 1998, 1485-1494.

[5] P. L. Holland, Acc. Chem. Res. 2008, 41, 905-914.

[6] C. J. Cramer, W. B. Tolman, Acc. Chem. Res. 2007, 40, 601608.

[7] D. J. Mindiola, Acc. Chem. Res. 2006, 39, 813-821.

[8] H. W. Roesky, S. Singh, V. Jancik, V. Chandrasekhar, Acc. Chem. Res. 2004, 37, 969-981.

[9] D. J. Mindiola, Angew. Chem. Int. Ed. 2009, 48, 6198-6200.

[10] J. Wu, T.-L. Yu, C.-T. Chen, C.-C. Lin, Coord. Chem. Rev. 2006, 250, 602-626.

[11] C. A. Wheaton, P. G. Hayes, B. J. Ireland, Dalton Trans. 2009, 4832-4846.

[12] N. Ajellal, J.-F. Carpentier, C. Guillaume, S. M. Guillaume, M. Helou, V. Poirier, Y. Sarazin, A. Trifonov, Dalton Trans. 2010, 39, 8363-8376.

[13] M. J. Stanford, A. P. Dove, Chem. Soc. Rev. 2010, 39, 486494.
[14] S. D. Ittel, L. K. Johnson, M. Brookhart, Chem. Rev. 2000, 100, 1169-1204.

[15] A. Nakamura, S. Ito, K. Nozaki, Chem. Rev. 2009, 109, 5215-5244.

[16] B. P. Carrow , K. Nozaki Macromolecules 2014, 47, 25412555.

[17] J. L. Rhinehart, N. E. Mitchell, B. K. Long, ACS Catal. 2014, 4, 2501-2504.

[18] C. N. Ayala, M. H. Chisholm, J. C. Gallucci, C. Krempner Dalton Trans. 2009, 9237-9245.

[19] J. Feldmann, S. McLain, A. Parthasarathy, W. J. Marshall, J. C. Calabrese, S. D. Arthur, Organometallics 1997, 16, 15141516.

[20] a) K. Landolsi, M. Rzaigui, F. Bouachir, Tetrahedron Lett. 2002, 43, 9463-9466.

[21] M. Tanabiki, Y. Sunada, H. Nagashima, Organometallics 2007, 26, 6055-6058.

[22] V.C. Annibale, R. Tan, J. Janetzko, L.M. Lund, D. Song, Inorg. Chim. Acta 2012, 380, 308-321.

[23] C.-H. Lin, H. Pan, V. N. Nesterov, M. G. Richmond, J. Organomet. Chem. 2013, 748, 56-62.

[24] K. Landolsi, F. Richard, F. Bouachir, J. Organomet. Chem. 2005, 690, 513-518.

[25] G. Bai, P. Wei, A. Das, D. W. Stephan, Organometallics 2006, 25, 5870-5878.

[26] A. D. Phillips, G. Laurenczy, R. Scopelliti, P. J. Dyson, Organometallics 2007, 26, 1120-1122.

[27] The ${ }^{1} \mathrm{H}$ and ${ }^{13} \mathrm{C}$ NMR as well as IR spectra of the starting $\beta$ diketimines and the $\beta$-diimine zinc complexes $\mathbf{1}$ and $\mathbf{2}$ are given in the electronic supplement.

[28] M. Cheng, D. R. Moore, J. J. Reczek, B. M. Chamberlain, E. B. Lobkovsky, G. W. Coates, J. Am. Chem. Soc. 2001, 123, 8738-8749.

[29] S. Yokota, Y. Tachi, S. Itoh, Inorg. Chem. 2002, 41, 13421344.

[30] E. Lobkovsky, priv. commun. 2004, CCDC 236294.

[31] R. Boese, D. Bläser, T. Eisenmann, S. Schulz, priv. commun. 2009, CCDC 730654.

[32] S. Schulz, T. Eisenmann, D. Bläser, R. Boese, Z. Anorg. Allg. Chem. 2009, 635, 995-1000.

[33] S. Schulz, T. Eisenmann, D. Schuchmann, M. Bolte, M. Kirchner, R. Boese, J. Spielmann, S. Harder, Z. Naturforsch. B 2009, 64, 1397-1400.

[34] S. Schulz, T. Eisenmann, U. Westphal, S. Schmidt, U. Flörke, Z. Anorg. Allg. Chem. 2009, 635, 216-220.

[35] S. Schulz, D. Schuchmann, U. Westphal, M. Bolte, Organometallics 2009, 28, 1590-1592.

[36] S. Schulz, T. Eisenmann, S. Schmidt, D. Bläser, U. Westphal, R. Boese, Chem. Commun. 2010, 46, 7226-7228.

[37] M. Biyikal, L. Lohnwitz, N. Meyer, M. Dochnahl, P. W Roesky, S. Blechert, Eur. J. Inorg. Chem. 2010, 1070-1081.

[38] S. Schulz, J. Spielmann, D. Bläser, C. Wölper, Chem. Commun. 2011, 47, 2676-2678.

[39] H.-Y. Chen, Y.-L. Peng, T.-H. Huang, A. K. Sutar, S. A Miller, C.-C. Lin, J. Mol. Catal. A 2011, 339, 61-71.

[40] G. Bendt, S. Schulz, J. Spielmann, S. Schmidt, D. Bläser, C. Wölper, Eur. J. Inorg. Chem. 2012, 3725-3731.

[41] All geometries were fully optimized using standard convergence criteria and standard integration grids at the density functional theory level, employing the B3-LYP exchange-correlation functional [a) A. D. Becke, Phys. Rev. A 1988, 38, 3098; b) J. P. Perdew, Phys. Rev. B 1986, 33 
8822.] as implemented in the Turbomole V6.3 quantum chemistry program package [a) TURBOMOLE V6.3 2011, a development of University of Karlsruhe and Forschungszentrum Karlsruhe $\mathrm{GmbH}, \quad$ 1989-2007, TURBOMOLE GmbH, since 2007; available from http://www.turbomole.com; b) R. Ahlrichs, M. Bär, M. Häser, H. Horn and C. Kölmel, Chem. Phys. Letters 1989, 162, 165; c) M. Häser and R. Ahlrichs, J. Comput. Chem. 1989, 10, 104; d) O. Treutler and R. Ahlrichs, J. Chem. Phys. 1995, 102, 346; e) M. v. Arnim and R. Ahlrichs, J. Chem. Phys. 1999, 111, 9183.] by using TmoleXClient 3.4 [C. Steffen, K. Thomas, U. Huniar, A. Hellweg, O. Rubner, A. Schroer, $J$ Comput. Chem. 2010, 31, 2967-2970.]. A quadruple -zeta valence quality Gaussian type function basis set termed def2TZVP [a) F. Weigend, F. Furche and R. Ahlrichs, J. Chem. Phys. 2003, 119, 12753; b) F. Weigend and R. Ahlrichs, Phys. Chem. Chem. Phys. 2005, 7, 3297.] has been used throughout The resolution-of-the-identity approximation was employed, making use of an appropriate auxiliary basis set [F. Weigend, Phys. Chem. Chem. Phys. 2006, 8, 1057.]. Atom coordinates and energies of the optimized geometry are given in the electronic supplement.

[42] M. Stender, R. J. Wright, B. E. Eichler, J. Prust, M. M. Olmstead, H. W. Roesky, P. P. Power, J. Chem. Soc. Dalton Trans. 2001, 3465-3469.

[43] G. M. Sheldrick, Acta Crystallogr. A 1990, 46, 467-473.

[44] G. M. Sheldrick, SHELXL-97, Program for the Refinement of Crystal Structures University of Göttingen, Göttingen (Germany) 1997. See also: G. M. Sheldrick, Acta Crystallogr. A 2008, 64, 112-122.

Received: ((will be filled in by the editorial staff)) Published online: ((will be filled in by the editorial staff)) 


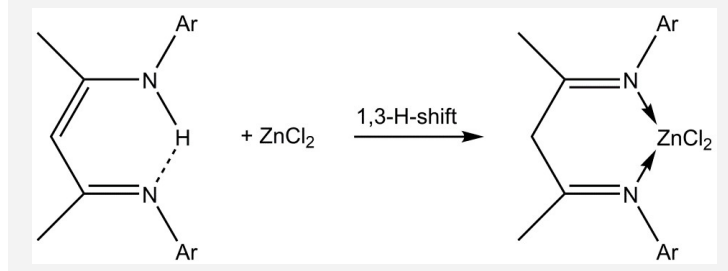

Christoph Scheiper, Dominik Naglav, Dieter Bläser, Christoph Wölper, Stephan Schulz* ........ Page No. - Page No.

Synthesis and X-ray crystal structures of zinc dichloride complexes supported by a $\beta$-diimine ligand 


\section{DuEPublico}

This text is made available via DuEPublico, the institutional repository of the University of Duisburg-Essen. This version may eventually differ from another version distributed by a commercial publisher.

DOI: $\quad 10.1002 /$ zaac. 201500046

URN: urn:nbn:de:hbz:464-20201125-110922-6

This is the peer reviewed version of the following article: Z. Anorg. Allg . Chem. 2015, 641, 871-875, which has been published in final form at: https://doi.org/10.1002/zaac.201500046 